\title{
Présence d'Asellides stygobies (Crustacea, Isopoda, Aselloidea) dans la région du Primorye, Sibérie sud-orientale
}

\author{
J.-P. Henry \& G. Magniez \\ Université de Bourgogne, Biologie Animale et Générale, 6, Bd. Gabriel, 21000 Dijon, France
}

Keywords: stygobiont asellids, taxonomy, biogeography, Far East and Pacific North America

\begin{abstract}
Two species of anophthalmous, unpigmented asellids have been found in springs and groundwaters of S.E. Siberia (Primorye region). Asellus (Asellus) primoryensis $\mathrm{n}$. sp. is closely related to the epigean species $A$. (A.) hilgendorfii Bovallius, 1886, as is the case for all stygobiont Asellus (Asellus) species previously known from the Japanese archipelago.

Sibirasellus parpurae n. g., n. sp. is closely related to the microphthalmous species Asellus dentifer Birstein \& Levanidov, 1952 from the Ussuri Basin (Khor region), now type-species of the new genus Sibirasellus. These two species show several original characters: body covered by numerous cuticular squamulae, mandibular palp reduced (glabrous and 2-segmented), and coxopodites of pereopods reduced and coalescent with their sternites, pointing to certain affinities with the stygobiont Japanese genus Nipponasellus Matsumoto, 1962 and probably to the epigean species of the "latifrons" group of the genus Asellus, presently restricted to arctic Siberia and western Alaska.

The different asellid lines living in the Far East and Pacific North America are much more related with each other than with all other lines of the family. All these forms possess a copulatory system built on the "Asellus pattern": Endopodite of 2nd male pleopod with a spur-shaped basal apophysis or "processus calcariformis", an afferent spermatic opening with a labial spur or "processus cylindriformis", and 2nd exopodite segment with a tergal or "catch lobe". This phyletic system includes the genus Asellus Geoffroy, 1762 (the present status of which is discussed herein), its subgenera Asellus Dudich, 1925, Mesoasellus Birstein, 1951, and Phreatoasellus Matsumoto, 1962, and its related genera Calasellus Bowman, 1981, Nipponasellus Matsumoto, 1962, Uenasellus Matsumoto, 1962, and Sibirasellus n. g.
\end{abstract}

\section{Résumé}

Deux espèces d'Asellides dépigmentés et anophtalmes ont été découvertes dans les eaux souterraines et sources de Sibérie sud- orientale (région du Primorye). L'espèce nouvelle Asellus (Asellus) primoryensis est étroitement apparentée à l'espèce épigée $A$. (A.) hilgendorfii Bovallius, 1886, comme c'était déjà le cas pour tous les Asellus (Asellus) stygobies précédemment découverts dans les eaux souterraines de l'archipel japonais.

L'espèce Sibirasellus parpurae n. g., n. sp. est étroitement apparentée à une espèce microphtalme du bassin de l'Oussouri, région de Khor: Asellus dentifer Birstein \& Levanidov, 1952 qui devient l'espèce-type du nouveau genre Sibirasellus. Ces deux espèces présentent plusieurs caractères originaux: corps couvert de nombreuses squamules cuticulaires, palpe mandibulaire glabre et réduit à 2 articles, et coxopodites des péréiopodes réduits et ankylosés avec leur sternite. Elles montrent ainsi des affinités certaines avec les Nipponasellus Matsumoto, 1962 des eaux souterraines du Japon et peut-être avec les espèces épigées du groupe "latifrons" du genre Asellus s. str., groupe actuellement cantonné à la Sibérie arctique et à l'Alaska occidental.

Les différentes lignées phylétiques d'Asellidae de l'ExtrêmeOrient boréal et de la région pacifique de l'Amérique du Nord sont davantage apparentées entre-elles qu'avec toutes les autres lignées de la famille. Elles possèdent toutes un appareil copulateur construit sur le "schéma Asellus": Endopodite des pléopodes II mâles avec une apophyse basale interne ou "processus calcariformis", un orifice spermatique afférent muni d'un "processus cylindriformis", (= "labial spur") et article 2 de l'exopodite avec un lobe tergal ou "catch lobe". Cet ensemble phylétique comprend le genre Asellus Geoffroy, 1762 (dont le statut actuel est discuté dans ce travail), ses sous-genres Asellus Dudich, 1925, Mesoasellus Birstein, 1951, et Phreatoasellus Matsumoto, 1962, et ainsi que les genres apparentés à Asellus: Calasellus Bowman, 1981, Nipponasellus Matsumoto, 1962, Uenasellus Matsumoto, 1962 et Sibirasellus n. g.

\section{Introduction}

A la suite de prospections effectuées par des chercheurs de l'Institut de Biologie et Pédologie de 
l'Académie des Sciences de Russie (Filiale de l'Extrême-Orient, à Vladivostok), une collection de 10 tubes (notés ici S1 à S10), contenant des Asellidae anophtalmes et dépigmentés des eaux souterraines et sources de la partie orientale du bassin de l'Oussouri, nous a été communiquée pour étude.

Nous remercions très vivement le Dr. I.M. Levanidova (Vladivostok), le Dr. L. Botosaneanu et le Prof. J.H. Stock (Amsterdam), pour la confiance qu'ils nous témoignent en nous confiant ce matériel comportant deux espèces nouvelles.

\section{Taxonomie}

Asellus (Asellus) primoryensis n. sp.

(Figs. 1-10, Pl. I)

Matériel examiné. - Un lot de tubes numérotés S1 à S8 et S10. Numéro de collection pour tous ces prélèvements: Zoölogisch Museum Amsterdam (ZMA) Isop. 105528.

S1 - "Primorye Lasovskyi reserve (P.L.r.); Imbishi River (subterranian)": 66 individus, dont 60 entiers (la majorité ont perdu leurs antennes et leurs uropodes): 23 mâles adultes de 5,2 à $8,5 \mathrm{~mm}$ (holotype: un mâle de $7,6 \mathrm{~mm}$ ), 35 femelles adultes de 5 à $9 \mathrm{~mm}$ dont 3 ovigères et la plupart des autres avec des oostégites de repos sexuel (allotype: une femelle de $7 \mathrm{~mm}$ ) et 2 jeunes de 1,5 mm. "Vshivkova col., 24/06/1979".

S2 - "P.L.r.; Imbishi River (subterranian); temp. 6,5 $5^{\circ}$; Vshivkova col., 11/06/1979": 1 mâle de 6,5 mm (avec uropodes de $3,1 \mathrm{~mm}$ ) et 1 femelle à oostégites réduits de $9 \mathrm{~mm}$.

S3 - “P.L.r.; Sukhoy Spring (subterranian); temp. $12,5^{\circ} \mathrm{C}$; Vshivkova col., 21/06/1979": 1 mâle de 4,8 mm.

S4 - "P.L.r.; Perekatnaya River basin; temp. 5,5 ${ }^{\circ}$; Vshivkova col., 30/05/1979'": 1 femelle à très petits oostégites de 5,8 $\mathrm{mm}$ (uropodes de $2,6 \mathrm{~mm}$ ).

S5 - “P.L.r.; Blisky Spring; Zapolina col., 02/06/1979": 1 femelle sans oostégites de $5,1 \mathrm{~mm}$ (antennes de $5 \mathrm{~mm}$ et uropodes de $2,2 \mathrm{~mm}$ ).

S6 - "P.L.r.; Imbishi River; temp. $7,5^{\circ} \mathrm{C}$; Vshivkova col., 10/06/1979': 1 mâle de 3,3 mm.

S7 - "P.L.r.; Bolotny Spring; temp. 10,0 ${ }^{\circ} \mathrm{C}$; Vshivkova col., 07/06/1979': 4 mâles de 6,2; 5,8; 4,8 et 4,4 mm; 3 femelles de 6; 4,5 et 3,9 mm, plus un Amphipode stygobie.

S8 - "Primorye; Bikin River; temp. 13,0 $0^{\circ}$; Timoshkin col.,
05/08/1979": 1 mâle de $6 \mathrm{~mm}$ (antennes de $6 \mathrm{~mm}$ également).

S10 - “Taukhe River; Semenchenko coll. 28/05/1980": 1 mâle de $6 \mathrm{~mm}$ et 3 femelles à oostégites réduits de 8,8 (uropodes de $3,3 \mathrm{~mm}), 8,0$ et $5,4 \mathrm{~mm}$.

Description. - Corps robuste (Fig. 1), totalement dépigmenté (d'un blanc pur dans l'alcool) et anophtalme. Forme allongée (coefficient d'allongement voisin de 4,5). Pléotelson quadrangulaire (longueur/largeur $=1,25$ ), portant de très longs uropodes.

Appendices céphaliques: Antennules plus longues que le céphalon, avec hampe de 4 et fouet de 13 articles portant tous, sauf les 2 proximaux et le dernier, une lame olfactive de $75 \mu \mathrm{m}$ de long chez le mâle de 7,6 $\mathrm{mm}$. Le fouet de l'antennule de la femelle de $7 \mathrm{~mm}$ ne porte que 9 articles et 6 lames olfactives. Antennes très longues, avec hampe de 6 articles et fouet de 72 pour une femelle de 7,5 $\mathrm{mm}$. Mandibules, maxillules et maxilles sans particularités notables (très semblables à celles d'Asellus (A.) hilgendorfii Bovallius, 1886); palpe mandibulaire normal à 3 articles sétigères et lame interne de la maxillule (Fig. 2) avec 4 tiges plumeuses sur la marge distale, ce qui est caractéristique du sous-genre Asellus.

Maxillipèdes typiques d'Asellidae, avec un rétinacle de 4 crochets à l'endite. Chez la femelle ovigère, l'oostégite est représenté par un petit lobe portant 9-10 tiges plumeuses à direction caudale.

Péréiopodes de longueur croissante du I au VII; longueurs relatives à celle du péréiopode $\mathrm{II}: \mathrm{I}=0,81$; II $=1,00 ; \mathrm{VII}=1,57$. Pas de différenciation en crochet nuptial du péréipode IV. Dactylopodite avec 6-8 épines sternales au péréiopode I (Fig. 3) et seulement 2 épines très allongées et bien espacées aux autres péréiopodes. Chez les plus grands mâles, une épine supplémentaire apparaît aux péréiopodes $\mathrm{V}, \mathrm{VI}$ et VII.

Appendices abdominaux: Pléopodes I mâles (Fig. 4) très allongés, l'exopodite est 2 fois plus long

Figs. 1-10. Asellus (Asellus) primoryensis n. sp.: 1, habitus d'un mâle de 7,6 mm, échelle = 1 mm; 2, lame interne d'une maxillule du même, é $=100 \mu \mathrm{m} ; 3$, péréiopode I du même, é $=100 \mu \mathrm{m} ; 4$, pléopode I droit, face sternale, du même, é $=100 \mu \mathrm{m} ; 5$, pléopode II droit, face sternale, du même, $\hat{e}=100 \mu \mathrm{m} ; 6$, pléopode Il droit, face sternale, d'une femelle de $7 \mathrm{~mm}, \hat{e}=100 \mu \mathrm{m} ; 7$, pléopode III du mâle de $7,6 \mathrm{~mm}$, é $=300 \mu \mathrm{m} ; 8$, pléopode III de la femelle, é $=500 \mu \mathrm{m} ; 9$, pléopode IV du mâle, é $=300 \mu \mathrm{m} ; 10$, pléopode $\mathrm{V}$ du même, é $=300 \mu \mathrm{m}$. 
Bijdragen tot de Dierkunde, 62 (3) - 1993

181

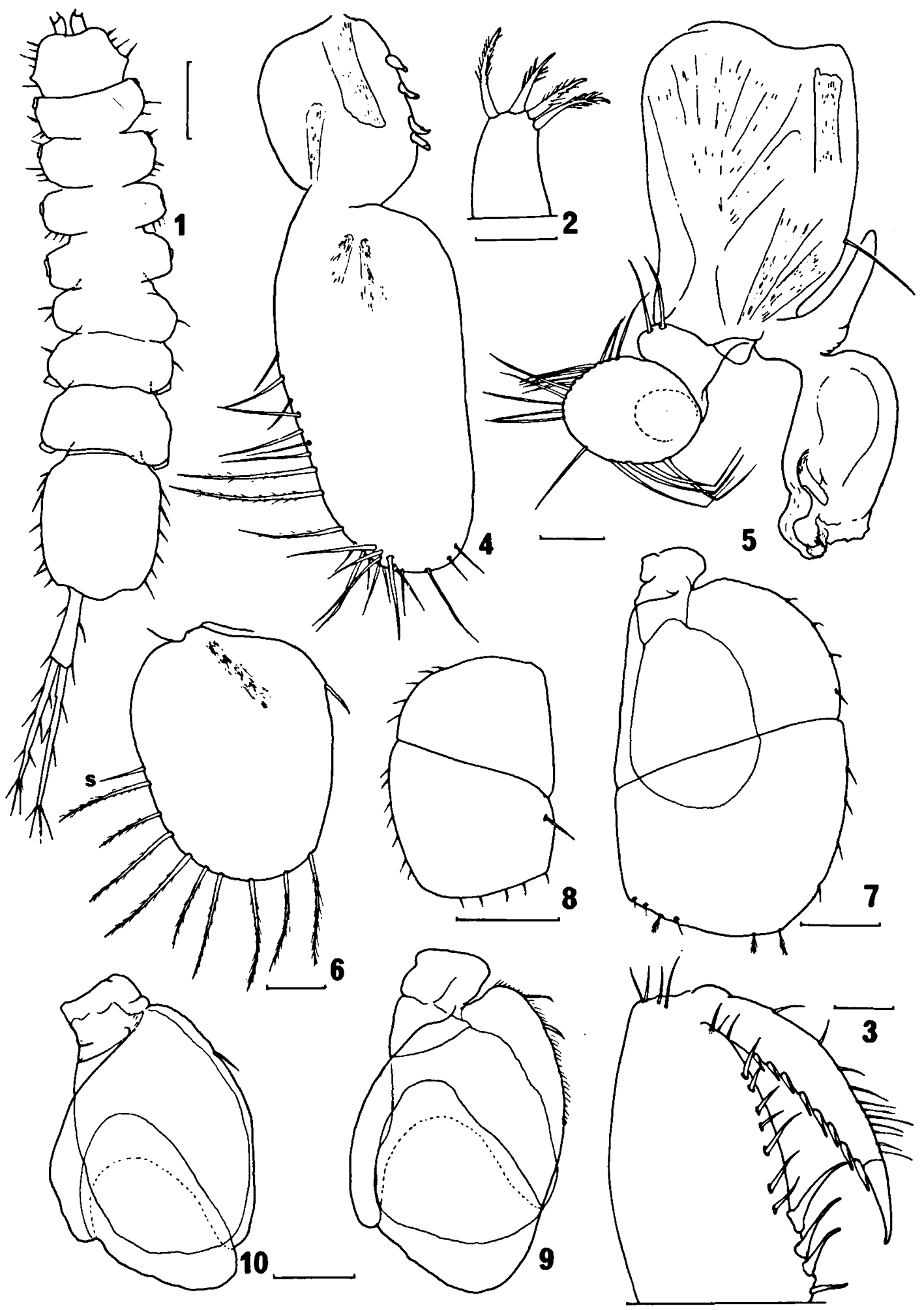

Downloaded from Brill.com04/26/2023 01:35:21PM

via free access 


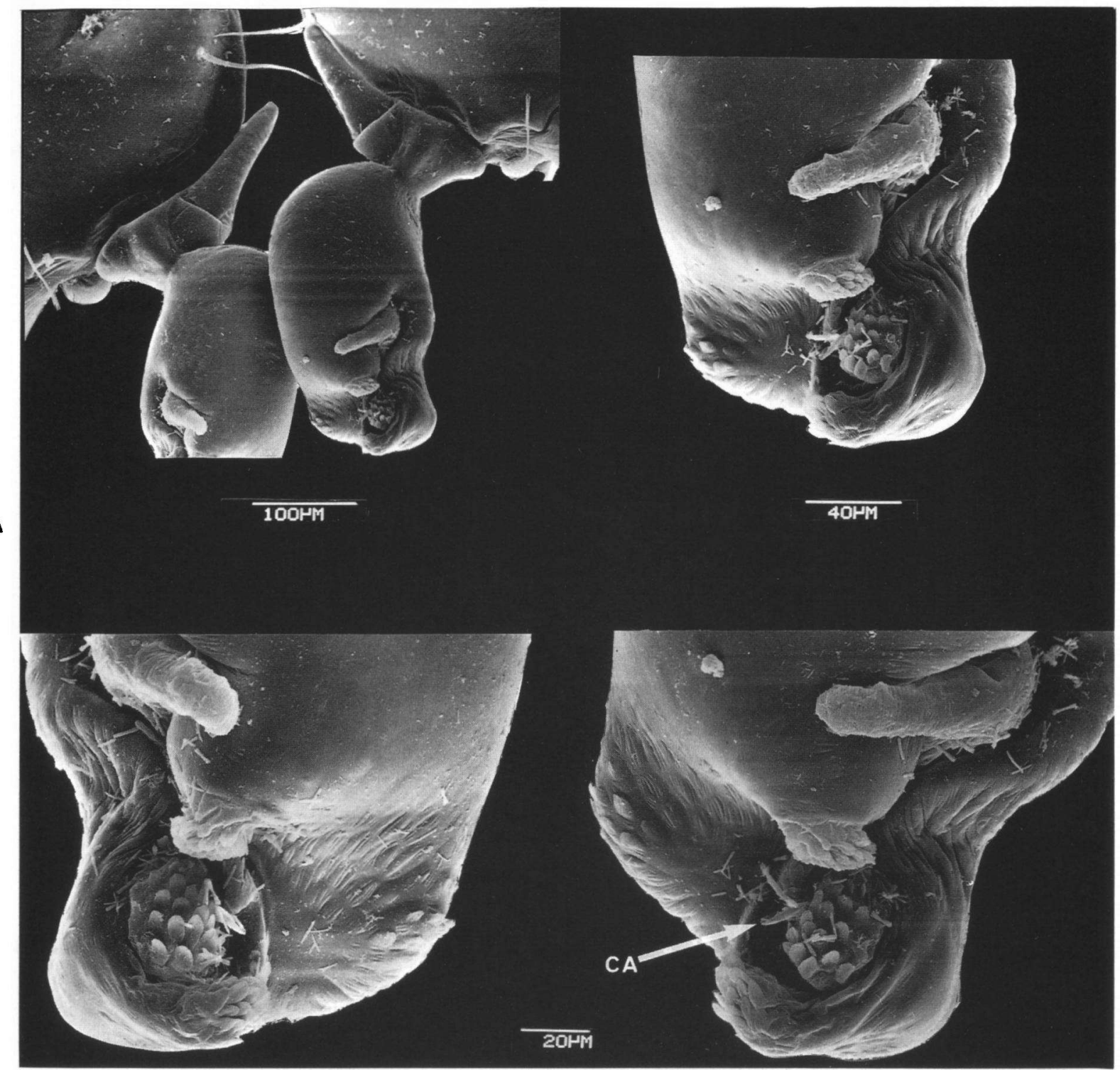

C

Planche I. Asellus (Asellus) primoryensis n. sp., clichés SEM des endopodites des pléopodes II d'un mâle. Noter en A la présence d'un sillon et d'une aire articulaire sur l'apophyse proximale ou "processus calcariformis", indiquant que cette apophyse représente vraisemblablement l'article distal originel de l'endopodite, le corps de l'organe copulateur n'étant sans doute qu'une expansion de l'article proximal. En B, le "processus cylindriformis" et le lobe antérieur, garni d'écailles épicuticulaires sont visibles. En C, le lobe antérieur, la membrane squamifère fermant la crypte du capitulum et la petite canule apparaissent. En D, la canule (CA) est notée. L'aspect de toutes ces structures est caractéristique des véritables "Asellus" (sous-genre Asellus).

que le protopodite; celui-ci porte à son bord interne 4 crochets rétinaculaires assez robustes. L'exopodite, 2 fois plus long que large, avec une série de 20-24 soies très inégales sur la marge externe: les plus apicales ont une insertion submarginale tandis que les plus longues sont plumeuses. Pléopodes II mâles (Fig. 5, Pl. I): aspect général caractéristique du genre Asellus. Structure de l'endopodite très voisine de celle d' $A$. hilgendorfii. On observe en particulier la présence d'une petite canule (Pl. I, C 
et $\mathrm{D}$ : $\mathrm{Ca}$ ), qui s'insère dans une crypte formée par la partie distale écailleuse du corps de l'endopodite (capitulum).

Ainsi, la seule différence notable entre l'espèce épigée et l'hypogée est la forme du protopodite, beaucoup plus allongé chez $A$. primoryensis n. sp. ( 1,5 fois plus long que large) que chez $A$. hilgendorfii (longueur pratiquement égale à la largeur). Le cliché SEM (Pl. I A) montre nettement que l'apophyse basale interne de l'endopodite ou "processus calcariformis" présente un sillon transversal jouxtant une aire de cuticule souple. L'existence de ces structures articulaires et la position du muscle endopodial permettent de penser que le "processus calcariformis" pourrait être l'article distal (article 2) de l'endopodite. Dans cette hypothèse, la partie copulatrice vésiculeuse de l'endopodite ne serait qu'un diverticule hypertrophié de l'article 1 et la morphologie de l'organe copulateur des Asellidae du groupe "Asellus" (Figs. 27 et 28), serait radicalement différente de celle des autres Asellidae, comme Baicalasellus, Bragasellus, Proasellus, Salmasellus, Synasellus, etc. (cf. le paragraphe "Discussion et conclusions").

Pléopodes II femelles subovalaires (Fig. 6); les bords internes des deux appendices se recouvrent légèrement. Une série de 10 tiges, toutes plumeuses, garnit les marges externe et distale et une petite soie exopodiale lisse (S) est présente; une autre, très semblable à la précédente se développe près de l'angle proximal interne de l'appendice.

Pléopodes III (Fig. 7) avec exopodite subovalaire, divisé en deux articles par une suture oblique; 10-12 courtes soies simples sont espacées sur la marge externe et 3 à 6 courtes et plumeuses sur la marge distale. Chez la femelle (Fig. 8), on note la présence constante d'une assez forte tige lisse sur la marge interne de l'article distal. Endopodite ovalaire, charnu, un peu plus long que l'article proximal de l'exopodite.

Pléopodes IV et V (Figs. 9, 10) avec exopodite montrant une disposition de l'aire charnue respiratoire très caractéristique du sous-genre Asellus.

Uropodes très allongés, presque 2 fois plus longs que le pléotelson (Fig. 1); leur longueur a été donnée dans le paragraphe "Matériel examiné" pour les rares individus qui les ont conservés.
Affinités. - Cette nouvelle espèce stygobie appartient sans erreur possible à la lignée de l'espèce superficielle $A$. (Asellus) hilgendorfii, de même que les nombreuses espèces dépigmentées et anophtalmes du même sous-genre Asellus mises en évidence dans les eaux souterraines de l'archipel nippon (Matsumoto, 1963, 1966, 1976; cf. Henry et al., 1986). Les clichés réalisés au SEM (Pl. I A à I C cijointes et Figs. 1 à 5 de Henry \& Magniez, 1991), de même que les Figs. 5 et 27-28, montrent la très grande similitude morphologique entre l'organe copulateur d' $A$. ( $A$.) hilgendorfii et celui de la nouvelle espèce. On peut donc en déduire qu'il existe une filiation directe entre l'espèce pigmentée-oculée et la forme stygobie décrite ici.

Ecologie et biogéographie. - Les terres émergées actuelles entourant la Mer du Japon (Corée et S.E. de la Sibérie, Sakhaline et totalité de l'archipel japonais), ont été, à de multiples reprises, reliées entre elles dans le Pliocène et lors des épisodes régressifs Quaternaires (Birstein, 1970). Elles ont donc possédé des systèmes hydrographiques en commun et, de ce fait, présenté des faunes identiques d'Asellus des eaux douces de surface (représentées à la fois par le sous-genre Asellus s. str. et par le sous-genre Phreatoasellus). Le premier sous-genre est toujours très largement représenté en surface par l'espèce polymorphe $A$. ( $A$.) hilgendorfii (bassin de l'Amour-Oussouri, Corée, Japon, Mandchourie, Chine), tandis que le second ne l'est plus que par l'espèce obscuricole et microphtalme $A$. (P.) kawamurai Tattersall, 1921 (Japon méridional), mais tous deux comprennent de nombreuses espèces dépigmentées et anophtalmes, d'abord mises en évidence dans la partie insulaire de l'aire (surtout Honshu, Shikoku, Kyûshû) (Matsumoto, 1976), mais également présentes dans les portions continentales: $A$. (Phreatoasellus) joianus Henry \& Magniez, 1991, de Corée et maintenant $A$. (Asellus) primoryensis n. sp. du Primorye.

Genre Sibirasellus n. g.

Espèce-type - Asellus (Mesoasellus) dentifer Birstein \& Levanidov, 1952. 
Diagnose. - Corps à chétotaxie réduite, mais couvert de squamules, portions latérales des tergites thoraciques dilatées et coxopodites réduits, mandibule avec palpe biarticulé, lame interne des maxillules avec 5 tiges ciliées distales, propodite des péréiopodes I avec marge palmaire rectiligne, endopodite des pléopodes 2 mâles avec processus calcariformis court et massif, processus cylindriformis petit, capitulum armé d'épines et présentant une courte canule, exopodite des uropodes nettement réduit.

La nécessité de créer cette nouvelle entité générique sera discutée après la partie descriptive.

\section{Sibirasellus parpurae n. sp.}

(Figs. 11-26)

Matériel examiné. - S9 - "Primorye; Yedinka River dist.; Peretytchicha vil.; from the pit-hole (4 m depth); Parpura col., autumn 1989'": 1 mâle adulte de $9 \mathrm{~mm}$, en partie détérioré (holotype), les fragments d'un autre mâle adulte de 7-8 $\mathrm{mm}, 1$ femelle adulte à oostégites réduits de $9,3 \mathrm{~mm}$ (allotype) et un pullus sans péréiopodes VII (coll. no. ZMA Isop. 105 529).

Description. - Corps (Fig. 11) totalement dépigmenté (blanc nacré dans l'alcool) et anophtalme, portant très peu de soies mais largement recouvert de fines écailles épicuticulaires dont l'orientation générale est uniforme dans une aire restreinte, mais varie selon les régions considérées (largeur de ces squamules jusqu'à $20 \mu \mathrm{m}$ et hauteur jusqu'à $10 \mu \mathrm{m}$, leur marge libre curviligne étant souvent garnie de sétules, Fig. 21). Ce revêtement fait penser à celui d'un Poisson ou d'un Squamate! Des productions similaires ont été signalées chez des espèces du genre américain Lirceus Rafinesque, 1820, comme Lirceus lineatus (Say, 1818) = Mancasellus tenax (Smith, 1876) (cf. Racovitza, 1920).

La forme et l'aspect sont également très caractéristiques: corps allongé (longueur/largeur = 4,27 ), à bords très parallèles; céphalon subquadrangulaire, plus large que long (longueur/largeur = $1,30)$, avec deux faibles protubérances représentant les lobes postmandibulaires; péréionite I beaucoup plus large que le céphalon et encadrant celui-ci de ses deux pointes latérales antérieures, aspect rappelant celui de $S$. dentifer. A la face sternale du péréionite $\mathrm{V}$ de la femelle en intermue de repos sexuel, les deux orifices génitaux (non fonctionnels à ce stade) sont visibles sous forme de deux cicatrices cuticulaires longitudinales de $160 \mu \mathrm{m}$ environ de longueur et située à $150 \mu \mathrm{m}$ de la marge interne du coxopodite ipsilatéral. Pléonites I et II libres, rétrécis en largeur et formant un net pédoncule au pléotelson; ce dernier très régulièrement elliptique, sauf deux petites échancrures au niveau des uropodes et allongé (longueur/largeur $=1,31$ ).

Appendices céphaliques: Antennules avec hampe de 4 et fouet de 9-12 articles, tous, sauf le proximal, portant une lame olfactive de 90-95 $\mu \mathrm{m}$ de long. Antennes avec hampe de 6 et fouet pouvant compter 45 articles ou plus (Fig. 11). Mandibules (Fig. 14) au corps couvert de squamules, comme pour les autres appendices; processus inciseur à 4 dents acérées, plus épaisses à la mandibule gauche, lacinia mobilis de celle-ci bien développée, suivie d'une dizaine d'épines denticulées (11 à droite); lobe molaire très long, avec son plateau distal garni de sétules; palpe mandibulaire ne comportant que 2 articles (le second nettement réduit), portant quelques squamules, mais aucune soie. Maxillules avec lobe interne muni de 5 tiges ciliées distales et lobe externe avec une douzaine de tiges denticulées distales. Maxilles trilobées typiques des Asellidae, avec de nombreuses lames falciformes.

Maxillipèdes typiques des Asellidae, endite à 4-5 crochets rétinaculaires, palpe sétigère à 5 articles, épipodite quadrangulaire avec seulement 3 courtes soies lisses sur la marge externe.

Appendices thoraciques: Péréiopodes I-VII grêles, leurs coxopodites réduits à un étroit anneau sclérifié, ankylosé, sans épimère (Fig. 13). Les coxopodites I à IV de la femelle adulte en intermue de repos génital portent un oostégite subquadrangulaire bien développé (Fig. 22). Péréiopodes I à propodite non renflé, avec marge palmaire subrectiligne, sans processus saillant (Fig. 23); celui de la femelle est encore plus grêle, avec une marge palmaire légèrement concave. Dactylopodites II à VII avec un ongle simple et 2 épines marginales sternales (Fig. 15). Péréiopodes IV du mâle à peine modifiés.

Appendices abdominaux: Pléopodes I mâles (Fig. 16) avec protopodite plus long que large 

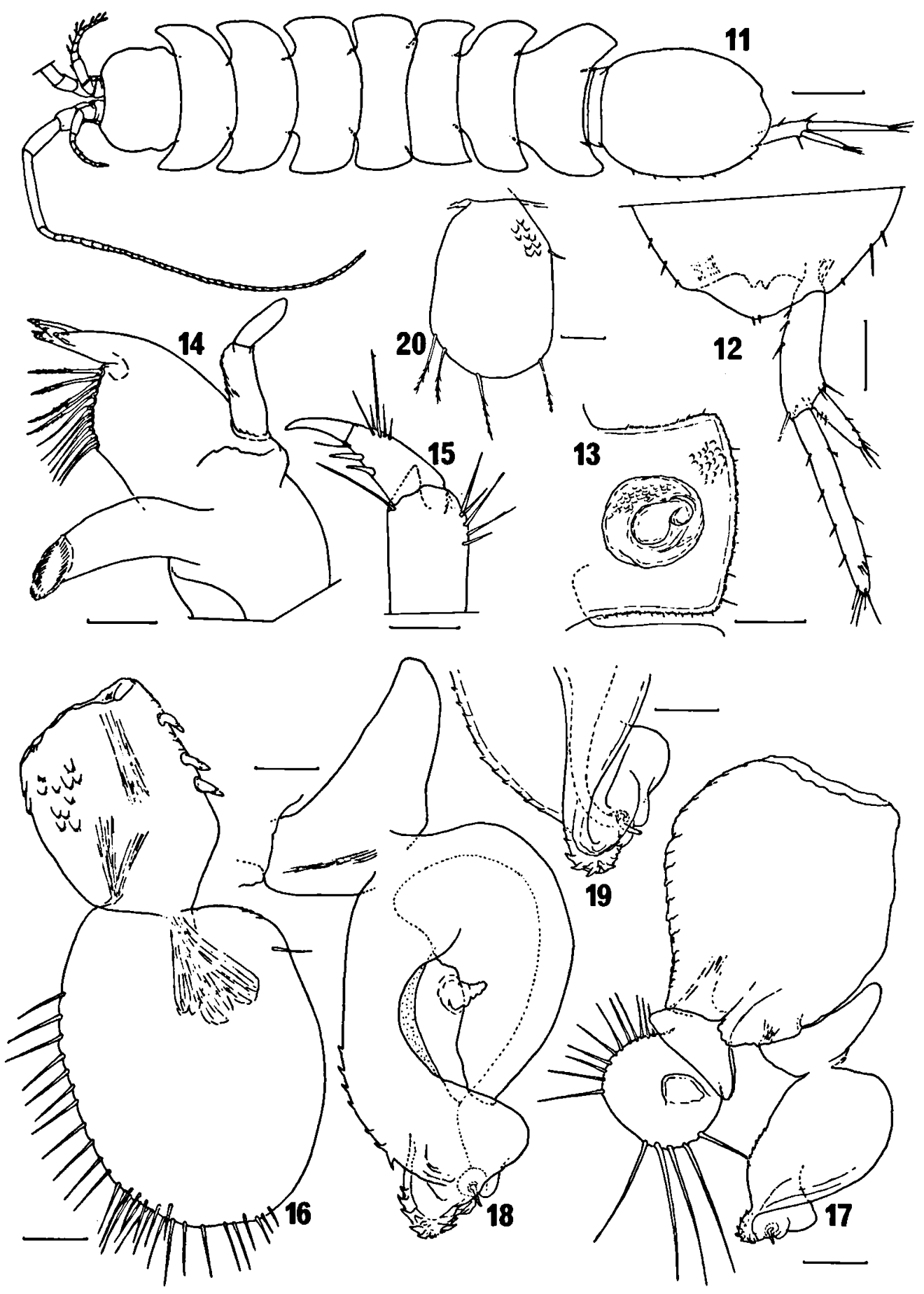

Figs. 11-20. Sibirasellus parpurae n. g., n. sp.: 11, habitus de la femelle de $9,3 \mathrm{~mm}$, échelle = $1 \mathrm{~mm} ; 12$, uropode droit du mâle de $9 \mathrm{~mm}, \hat{e}=200 \mu \mathrm{m} ; 13$, coxopodite IV gauche du mâle de $7-8 \mathrm{~mm}, \hat{e}=200 \mu \mathrm{m} ; 14$, mandibule droite du même, é $=100 \mu \mathrm{m} ; 15$, extrémité d'un péréiopode IV du même, é $=100 \mu \mathrm{m} ; 16$, pléopode I droit, face sternale, du même, é = $100 \mu \mathrm{m} ; 17$, pléopode II gauche, face tergale, du même, é $=100 \mu \mathrm{m} ; 18$, face sternale de l'endopodite du même, le petit "processus cylindriformis" est représenté, é $=50 \mu \mathrm{m} ; 19$, portion distale, face tergale, du même, la canule (base cachée en pointillés) est figurée, é $=50 \mu \mathrm{m} ; 20$, pléopode II droit, face sternale, de la femelle, $c=100 \mu \mathrm{m}$. 


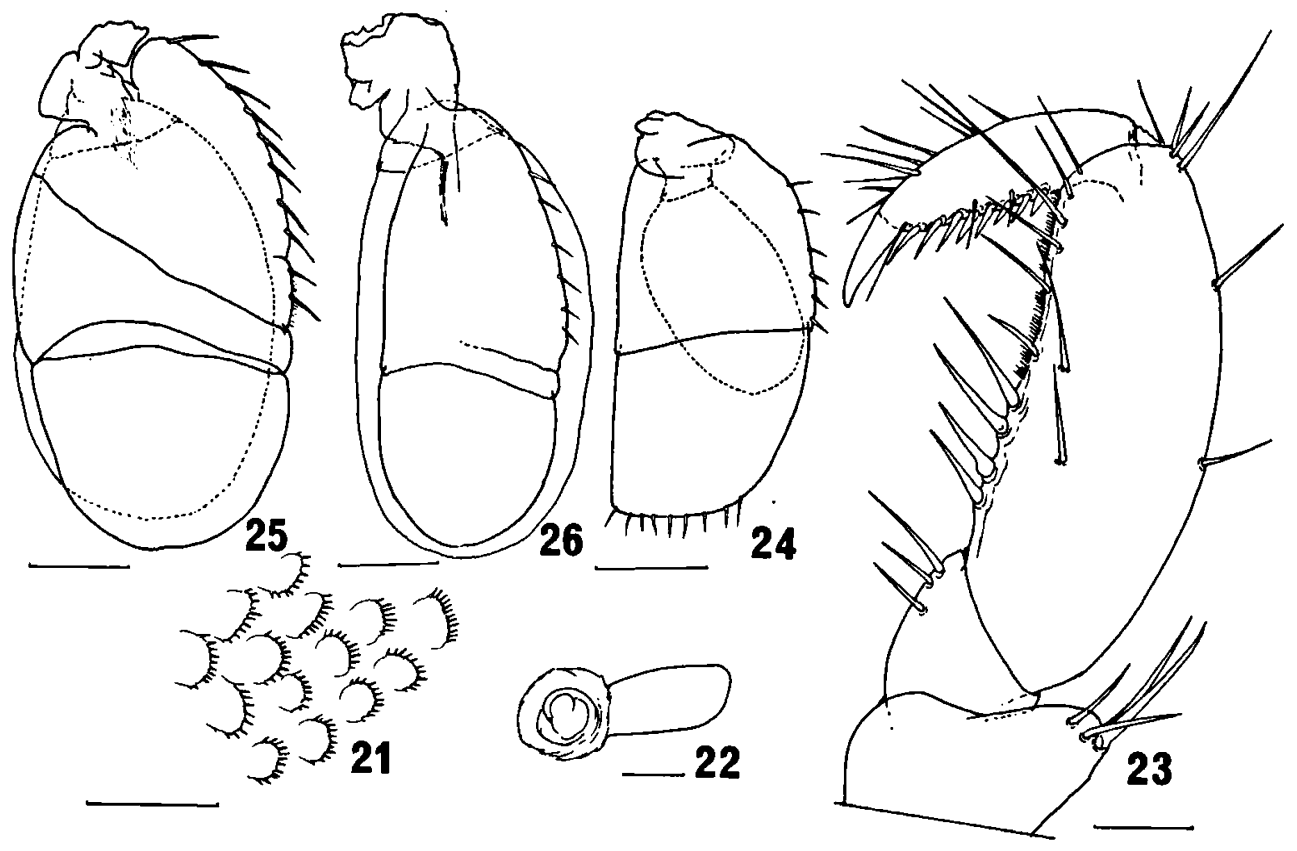

Figs. 21-26. Sibirasellus parpurae n. g., n. sp.: 21, écailles épicuticulaires d'un péréionite de la femelle, é $=50 \mu \mathrm{m}$; 22 , oostégite de la même, é $=200 \mu \mathrm{m} ; 23$, péréiopode I du mâle de $9 \mathrm{~mm}$, é $=100 \mu \mathrm{m} ; 24$, pléopode III gauche, face sternale, du même, é $=400 \mu \mathrm{m}$; 25, pléopode IV gauche, face sternale, du même, é $=200 \mu \mathrm{m} ; 26$, pléopode $\mathrm{V}$ gauche, face sternale, du même, é $=200 \mu \mathrm{m}$.

(longueur/largeur $=1,35$ ), partiellement écailleux, rétinacle à $2-3$ crochets; exopodite ovalaire large et très court (longueur/largeur $=1,22$ ), avec plus de 30 courtes soies marginales et submarginales.

Pléopodes II mâles (Fig. 17) avec protopodite allongé à marge externe écailleuse; exopodite court, en forme de gland avec article distal presque quadrangulaire, plus large que long, muni d'un lobe tergal (catch lobe) bien développé, et d'un groupe d'une dizaine de soies marginales à direction externe plus 5 fortes tiges dirigées vers l'endopodite, si bien que la marge distale est en partie nue. Endopodite (Fig. 18) avec apophyse basale interne (processus calcariformis ou basal spur) courte et massive; corps de l'endopodite fusiforme avec partie distale (capitulum) vrillée et fortement armée d'épines, orifice spermatique afférent occupant une bonne part de la face sternale, mais le processus cylindriformis ( = labial spur) est petit, comme chez les Asellus de type “latifrons" et les Nipponasellus; une petite canule est mieux visible par la face tergale et par transparence un conduit interne reliant la vésicule spermatique à sa base semble présent (Fig. 19).
Pléopodes II femelles subovalaires (Fig. 20), plus longs que larges (longueur/largeur $=1,45$ ), non contigus et portant seulement quelques soies marginales distales.

Pléopodes III (Fig. 24) avec exopodite allongé (longueur/largeur $=1,90$ ), les deux articles sensiblement de même longueur; quelques courtes soies sur la marge externe du proximal et la marge distale du second. Endopodite petit, régulièrement ovalaire, charnu et respiratoire.

Pléopodes IV (Fig. 25) avec exopodite ovalaire (longueur/largeur = 1,83); une dizaine de soies et des sétules intermédiaires sur la marge externe du premier article; linea transversalis partiellement visible, linea areae bien nette et aire charnue respiratoire distale bien développée. Endopodite charnu respiratoire régulièrement ovalaire.

Pléopodes V (Fig. 26) avec exopodite ovalaire plus étroit que le IV (longueur/largeur = 2,45); marge externe de l'article proximal avec une demidouzaine de courtes soies, linea conjungens non visible, linea duplex très partiellement visible mais linea articularis complète, délimitant une aire charnue distale. Endopodite régulièrement ovalaire, 
très semblable à l'endopodite IV, aussi long que l'exopodite, mais plus large.

Uropodes (Fig. 11) aussi longs que le pléotelson, presque glabres, mis à part le faisceau de soies terminales; exopodite beaucoup plus court que la rame interne; cet exopodite est encore plus court chez le mâle (exopodite/endopodite $=0,35$, Fig. 12).

Affinités. - La nouvelle espèce est étroitement apparentée à l'espèce microphtalme de sources du bassin de l'Oussouri, région à l'Est de Khor: Sibirasellus dentifer. Elles possèdent en commun des caractères tellement particuliers (aspect général du corps et forme des tergites, présence généralisée de squamules cuticulaires, coxopodites réduits, palpe mandibulaire biarticulé et quasi glabre, structure des pléopodes I et II mâles, en particulier réduction $\mathrm{du}$ "processus cylindriformis" de l'endopodite II, structure des exopodites des pléopodes IV et $\mathrm{V}$, réduction de l'exopodite des uropodes) qu'il convient d'en faire une unité systématique particulière: Sibirasellus n. g.

Certains de ces caractères rappellent l'aspect que l'on observe dans d'autres lignées, d'une part chez les Asellus épigés de la lignée arctique "latifrons" (réduction des coxopodites des péréiopodes et expansion corrélative des marges latérales des tergites, architecture générale de l'organe copulateur), et d'autre part chez les Nipponasellus anophtalmes de l'archipel japonais (réduction du palpe mandibulaire, expansion des marges latérales des tergites) (Matsumoto, 1966).

Il serait intéressant d'effectuer des prospections systématiques dans toute la région du bassin de l'Amour, afin de définir l'aire de répartition de $S$. dentifer et de $S$. parpurae n. sp. et aussi de préciser si cette intéressante lignée des Sibirasellus n. g. est représentée par d'autres formes stygobies.

\section{Discussion et conclusions}

Des deux espèces stygobies décrites ici, la première, Asellus (Asellus) primoryensis n. sp. montre une filiation directe avec une grande espèce épigée, $A$. (A.) hilgendorfii Bovallius, 1886, qui est elle-même un Asellus typique. Il est donc normal de l'attribuer au genre Asellus des auteurs car, en cas de révision taxonomique ultérieure, "primoryensis" et "hilgendorfii" resteront, à l'évidence, unis dans la même ensemble de niveau générique.

Les caractères montrés par Sibirasellus parpurae n. sp. sont au contraire si particuliers qu'il nous semble nécessaire de créer un nouveau genre. Une autre raison doit être invoquée pour justifier cette création: c'est le caractère imprécis de la définition actuelle du "genre Asellus", dont nous résumons ci-dessous l'évolution des contours. Cette diagnose a été donnée en détail par Birstein (1951) et Matsumoto $(1962,1963)$; elle ne comporte que des caractères généraux propres aux Asellidae ou répandus chez les Aselloidea ou même les Asellota.

Le genre Asellus a été créé en 1762 par E.L. Geoffroy (Etienne-Louis Geoffroy, 1725-1810 et non Etienne Geoffroy Saint-Hilaire 1771-1848!; cette erreur que l'on trouve déjà dans les travaux de Sars, 1897, revient ensuite dans toute la littérature asellidienne; remarquons cependant que des auteurs américains anciens, comme De Kay, 1844, attribuent bien la paternité du genre à Geoffroy), pour l'unique espèce Oniscus aquaticus Linnaeus, 1758. De ce fait, la définition d'Asellus se confondait avec celle de la famille Asellidae Rafinesque, 1815 ( = Asellidae Sars, 1897). Par la suite, de nombreux auteurs eurasiatiques ont décrit des espèces oculées ou anophtalmes d'Asellidae en les attribuant toutes sans discussion au genre Asellus.

Le premier essai de mise en ordre taxonomique fut tenté par Dudich (1925), avec la création des deux sous-genres Asellus, pour A. aquaticus (L., 1758) et Proasellus, pour les Asellidae du bassin méditerranéen dont le type est $P$. meridianus (Racovitza, 1919).

La seconde tentative fut celle de Stammer (1932), qui définit les quatre sous-genres Asellus (pour $A$. aquaticus et les Asellidae d'Extrême-Orient, dont A. hilgendorfii Bovallius, 1886), Proasellus (pour $P$. meridianus (Racovitza, 1919) et espèces apparentées d'Europe occidentale et régions périméditerranéennes), Baicalasellus (pour des espèces originales du lac Baikal) et enfin Conasellus, pour les espèces d'Amérique du Nord (bien que des genres particuliers aient été définis antérieurement 


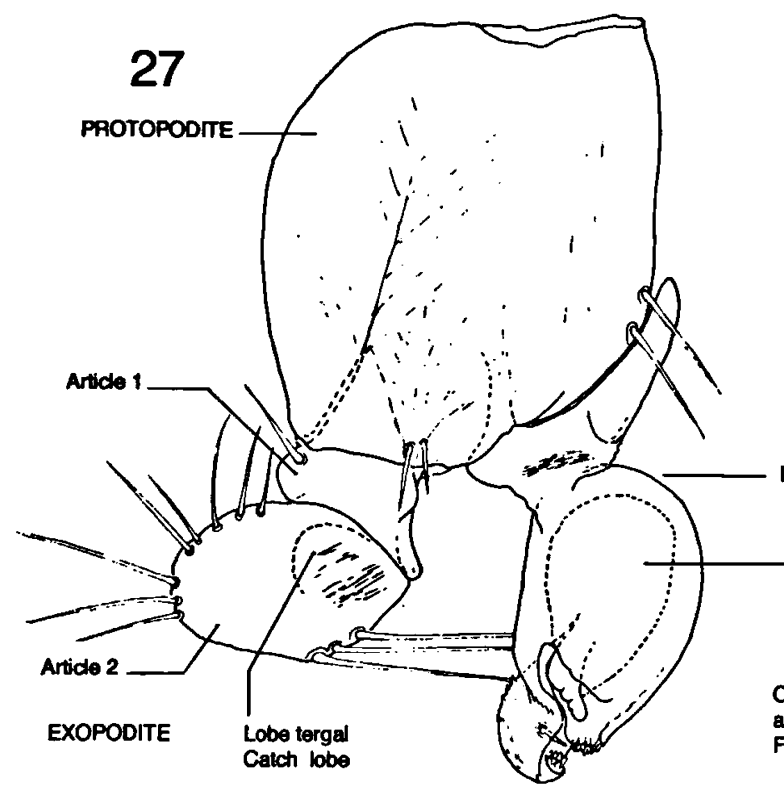

Figs. 27-28. Asellus (Asellus) hilgendorfii Bovallius, 1886 de l'île Popova, près Vladivostok, pléopode II mâle: 27, face sternale du pléopode entier; 28 , face sternale de son endopodite.

pour ce continent: Lirceus Rafinesque, 1820 et Caecidotea Packard, 1871, certaines espèces oculées y avaient en effet été décrites comme Asellus!).

Le cadre traditionnel du "genre Asellus" est utilisé pour la dernière fois par Birstein (1951), qui est obligé d'y distinguer: le sous-genre Asellus de Dudich et de Stammer, le sous-genre nouveau Mesoasellus Birstein, réunissant une espèce du Baïkal et des espèces nipponnes, le sous-genre Baicalasellus Stammer, le sous-genre Proasellus Dudich et le sous-genre Conasellus Stammer. Après cette date, et malgré la qualité exceptionnelle du travail de Birstein, la systématique des Asellidae est devenue difficile à interpréter, avec la survie du genre polyphylétique "Asellus" et la prolifération des espèces nouvelles.

Le premier auteur à rechercher une clarification de cette systématique, redéfinissant le genre comme un ensemble d'espèces plus apparentées entre elles qu'avec toutes les autres espèces de la famille et constituant une unité génétique et biogéographique indiscutable fut Braga (1944), avec la création du

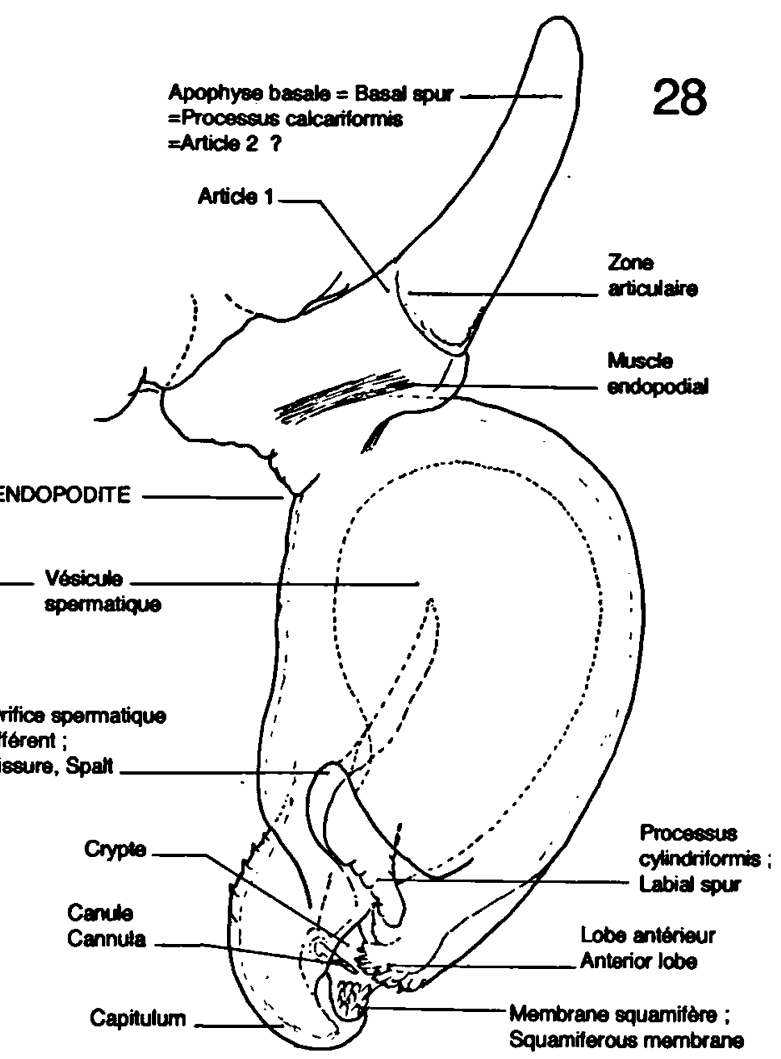

genre lusitanien Synasellus, groupe homogène de nombreuses espèces d'Asellidae stygobies, propre à la péninsule ibérique.

Les auteurs ultérieurs ont cherché à définir les véritables groupes évolutifs naturels d'espèces d'Asellidae en utilisant au maximum le cadre générique:

Matsumoto (1962) crée les genres Nipponasellus et Uenasellus pour une série d'espèces stygobies du Japon qui, autrement, auraient été incluses dans le cadre traditionnel du genre Asellus des auteurs; il distingue également un sous-genre particulier d'Asellus: Phreatoasellus, pour des espèces stygophiles ou stygobies au corps très longiligne.

Henry \& Magniez $(1968,1970)$ élèvent au rang de genre les sous-genres Proasellus (qui est devenu entre-temps un énorme ensemble périméditerranéen d'espèces, comprenant lui-même des lignées différentes) et Baicalasellus Stammer. Ils créent un genre nouveau Bragasellus pour désigner une lignée asellidienne propre à la péninsule ibérique. Les découvertes ultérieures n'ont fait que confirmer la 
validité de cette lignée ibérique (Henry \& Magniez, 1989). L'existence en Europe de deux espèces stygobies présentant chacune des caractères très particuliers devait également amener la création de deux nouvelles entités génériques monospécifiques: Gallasellus Henry \& Magniez, 1977 et Chthonasellus Argano \& Messana, 1991.

Grâce à la clairvoyance de Th.E. Bowman, l'existence de lignées évolutives naturelles indépendantes (et différentes des lignées eurasiatiques), au sein des Asellidae du Nouveau Monde a été également mise en exergue avec, à côté des genres multispécifiques classiques Lirceus Rafinesque, 1820 et Caecidotea Packard, 1871 (comportant l'un et l'autre des espèces oculées et des anophtalmes), la création de genres paucispécifiques ou monospécifiques particuliers: Salmasellus Bowman, 1975; Lirceolus Bowman \& Longley, 1976; Calasellus Bowman, 1981 et Remasellus Bowman \& Sket, 1985.

Ce passage d'une systématique statique à une systématique évolutive a eu l'avantage de bien définir (en faisant souvent appel à la morphologie comparée des pléopodes sexuels) les genres les plus récemment reconnus, qui sont homogènes, mais l'inconvénient de laisser subsister un doute sur le contenu et la validité phylétique du genre Asellus des auteurs. Parmi les sous-genres d'Asellus définis par Birstein en 1951, le sous-genre Mesoasellus se trouve réduit à la seule espèce $A$. (M.) dybowskii Semenkevich, 1924 (cf. Birstein, 1951), des profondeurs du lac Baïkal (toutes les autres ont été réunies dans le sous-genre Phreatoasellus Matsumoto, 1962 qu'il serait judicieux de considérer, dans le contexte taxonomique actuel, comme un genre à part entière). Il serait utile de réétudier en détail cette espèce abyssale du Baïkal ( $A$. (M.) dybowskii), qui présente des caractères exceptionnels (forme de l'habitus, des pléopodes III, des uropodes, etc.), afin de connaître ses affinités exactes. Pour le moment, il serait donc imprudent d'utiliser ce sousgenre Mesoasellus, aussi bien pour y laisser Sibirasellus dentifer (Birstein \& Levanidov, 1952) que pour y placer $S$. parpurae n. sp.

Le sous-genre Asellus s. str. correspond par contre à un grand ensemble comportant des espèces épigées à vaste répartition: $A$. aquaticus (Linnaeus, 1758), A. hilgendorfii Bovallius, 1886 et son impor- tant cortège d'espèces stygobies, plus la lignée arctique d'A. latifrons Birstein, 1947 (cf. Birstein, 1951). Des caractères morphologiques propres (caractères dignes de définir un genre!) le définissent: lobe interne de la maxillule avec 4 tiges pennées seulement, alors que le nombre de base est 5 chez les Asellidae, marge palmaire du propodite du péréiopode I du mâle armée d'une expansion à épines ensiformes, etc. C'est ce sous-genre Asellus s. str. qui devrait maintenant être élevé au rang de genre Asellus rénové (même dans cette optique, il resterait un problème phylétique à résoudre, car les relations entre les lignées "aquaticus-hilgendorfii", dont les coxopodites des péréiopodes sont libres et la lignée "latifrons", dont les coxopodites sont réduits à un anneau scléreux et soudés au sternite, sont loin d'être élucidées). Le genre Asellus auct. reste donc actuellement le "trou noir" de la systématique des Asellidae et mieux vaut simplifier la situation en n'y égarant pas les Sibirasellus.

Pour résumer le problème posé par les Asellidae originaires de la région nord-pacifique, on peut dire qu'il existe dans cette zone géographique un ensemble d'espèces d'origine commune, prouvée par l'unité architecturale des pléopodes II mâles: présence d'un lobe tergal accessoire ou "catch lobe" (assurant le maintien du "processus calcariformis"' lors de la copulation) à l'article distal de l'exopodite; présence d'un "processus calcariformis" ou "basal spur", à la base interne de l'endopodite (structure qui pourrait bien représenter l'article distal originel de cette rame, car une zone articulaire, en direction de laquelle s'oriente le muscle endopodial est nettement visible au SEM, à la base de ce processus: $\mathrm{Pl}$. I et Fig. 28); présence, au niveau de l'orifice spermatique afférent (ou "fissure"), d'un dispositif de fermeture comprenant un volet membraneux lié à un processus digitiforme dit "processus cylindriformis" ou "labial spur". Ces structures n'existent pas ou n'ont pas d'homologues chez les autres Asellidae mondiaux. Cet ensemble faunistique (qui mériterait d'être désigné taxonomiquement) correspond aux genres et sous-genres Asellus s. str. (Eurasie boréale et Alaska), Mesoasellus (Baïkal), Phreatoasellus (Japon, Corée), Nipponasellus (Japon), Uenasellus (Japon) et également Calasellus (Ouest de l'Amérique du Nord) qui, bien que 
profondément modifié, laisse paraître la même architecture de base que les précédents.

La création d'un genre Sibirasellus correspond à la nécessité de montrer l'existence d'un rameau supplémentaire original dans ce buisson évolutif dont la structure devra nécessairement se traduire bientôt par l'établissement d'un dendrogramme. Pour l'établir, une étude détaillée de la morphologie comparée des organes copulateurs par des moyens modernes (SEM) serait utile. Elle devrait porter au moins sur les espèces Asellus latifrons Birstein, 1947, A. epimeralis Birstein, 1947, Mesoasellus dybowskii (Semenkevich, 1924) (cf. Birstein, 1951, pour ces trois espèces), Phreatoasellus kawamurai (Tattersall, 1921), Sibirasellus dentifer (Birstein \& Levanidov, 1952), Nipponasellus aioii (Chappuis, 1955) (cf. Matsumoto, 1966) et Calasellus californicus (Miller, 1933) (cf. Bowman, 1981).

\section{Bibliographie}

Argano, R. \& G. Messana, 1991. A new stygobitic crustacean in the Ligurian Alps (Italy): Chthonasellus bodoni n. gen., n. sp. (Crustacea, Isopoda, Asellota). Stygologia, 6(4): 209-216.

Birstein, J.A., 1951. Asellota. Faune de l'U.R.S.S., 7(5): 1-142 (Akad. Nauk SSSR, Moscou) (en russe).

Birstein, J.A., 1970. Caractéristique zoogéographique de la faune souterraine de l'Union soviétique. In: T. Orghidan et al. (eds.), Livre du centenaire Emile G. Racovitza 1868-1968: 211-221 (Ed. Acad. Rép. soc. Roumanie, Bucarest).

Birstein, J.A. \& V. Ya. Levanidov, 1952. Une nouvelle espèce d'Aselle souterraine du bassin de l'Oussouri. C.r.Acad. Sci. URSS, 84(5): 1081-1084 (en russe).

Bovallius, C., 1886. Notes on the family Asellidae. Bih. K. svenska Vetensk. Akad. Handl., 11(15): 3-54.

Bowman, Th.E., 1975. Three new troglobitic asellids from western North America (Crustacea: Isopoda: Asellidae). Int. J. Speleol., 7: 339-356.

Bowman, Th.E., 1981. Calasellus longus, a new genus and species of troglobitic asellid from Shaver Lake, California (Crustacea: Isopoda: Asellidae). Proc. biol. Soc. Wash., 94(3): 866-872.

Bowman, Th.E. \& G. Longley, 1976. Redescription and assignment to the new genus Lirceolus of the Texas troglobitic water slater, Asellus smithii (Ulrich) (Crustacea: Isopoda: Asellidae). Proc. biol. Soc. Wash., 88(45): 489-496.

Bowman, Th.E. \& B. Sket, 1985. Remasellus, a new genus for the troglobitic swimming Florida asellid isopod, Asellus parvus Steeves. Proc. biol. Soc. Wash., 98(3): 554-560.

Braga, J.M., 1944. Note sur un genre d'Asellides nouveau du Portugal (Synasellus n. gen.) et description d'une sous-espèce nouvelle de ce genre. Mems. Estud. Mus. zool. Univ. Coimbra, 158: 1-11.

Dudich, E., 1925. Systematische Studien an italienischen Aselliden. Annls. hist.-nat. Mus. natn. hung., 22: 281-301.

Henry, J.-P. \& G. Magniez, 1968. Sur la systématique et la biogéographie des Asellides. C. r. hebd. Séanc. Acad. Sci., Paris, (D) 267: 87-89.

Henry, J.-P. \& G. Magniez, 1970. Contribution à la systématique des Asellides (Crustacea Isopoda). Annls. Spéléol., 25(2): 335-367.

Henry, J.-P. \& G. Magniez, 1977. Observations sur Gallasellus heilyi (Legrand, 1956), représentant d'un nouveau genre d'Asellide souterrain de France. Bull. Soc. zool. France, 102(2): 215-222, pls. I-II.

Henry, J.-P. \& G. Magniez, 1989. Isopodes Aselloïdes stygobies d'Espagne récoltés par J. Notenboom et I. Meijers. II - Le genre Bragasellus et description de sept nouvelles espèces. Stygologia, 4(4) “1988”': 332-362.

Henry, J.-P. \& G. Magniez, 1991. Quelques Asellotes stygobies et obscuricoles du Sud-Est de la Corée. Stygologia, 7(4): 217-225.

Henry, J.-P., J.J. Lewis \& G. Magniez, 1986. Isopoda: Asellota: Aselloidea, Gnathostenetroidoidea, Stenetrioidea. In: L. Botosaneanu (ed.), Stygofauna mundi: 434-464 (E.J. Brill/Dr. W. Backhuys, Leiden).

Kay, J.E. de, 1844. Zoology of New York or the New York fauna ..., part 6. Crustacea: 1-70, pls. 1-13 (Carroll \& Cook, Albany).

Matsumoto, K., 1962. Two new genera and a new subgenus of the family Asellidae of Japan. Annotnes. 20ol. jap., 35: 162-169.

Matsumoto, K., 1963. Studies on the subterranean Isopoda of Japan with notes on the well-water fauna of Japan (part I). Annls. Tokyo-to Lab. med. Sci., 13: 1-76.

Matsumoto, K., 1966. Studies on the subterranean Isopoda of Japan with notes on the well-water fauna of Japan (part II). Annls. Tokyo-to Lab. med. Sci., 23: 77-103.

Matsumoto, K., 1976. An introduction to the Japanese groundwater animals with reference to their ecology and hygienic significance. Int. J. Speleol., 8(1/2): 141-155.

Miller, M.A., 1933. A new blind isopod, Asellus californicus, and revision of the subterranean asellids. Univ. Calif. Publs. Zool., 39(4): 97-110.

Packard, A.S., 1971. The Mammoth Cave and its inhabitants. On the crustaceans and insects. Amer. Natural., 5: 744-761.

Racovitza, E.G., 1919. Notes sur les Isopodes. 1.-Asellus aquaticus auct. est une erreur taxonomique. 2.-Asellus aquaticus L. et A. meridianus n. sp. Archs. Zool. expér. gén., 58 (Notes Revue)(2): 31-43.

Racovitza, E.G., 1920. Notes sur les Isopodes. 8. Mancasellus tenax (Smith). 9. Mancasellus macrurus Garman. Archs. Zool. expér. gén., 59 (Notes Revue)(2): 28-66.

Rafinesque, C.S., 1820. Annals of nature or annual synopsis of new genera and species of animals, plants, etc., discovered in North America by C.S. Rafinesque. First annual number for 1820: 1-16 (Th. Smith ed., Lexington, $\mathrm{Ky}$.). 
Sars, G.O., 1897. An account of the Crustacea of Norway with short descriptions and figures of all the species. Vol. II. Isopoda. Part V, VI. Idotheidae, Arcthuridae, Asellidae, Ianiridae, Munnidae: 81-116, pls. 33-48 (Bergen Museum, Bergen).

Stammer, H.J., 1932. Zur Kenntnis der Verbreitung und Systematik der Gattung Asellus, insbesondere der mittel- europäischen Arten (Isopoda). Zool. Anz., 99: 113-131.

Tattersall, W.M., 1921. Zoological results of a tour in the Far East. Mysidacea, Tanaidacea and Isopoda. Mem. Asiat. Soc. Beng., 6(7): 403-433, [1-2], pls. XV-XVII.

Reçu le 23 mai 1992 